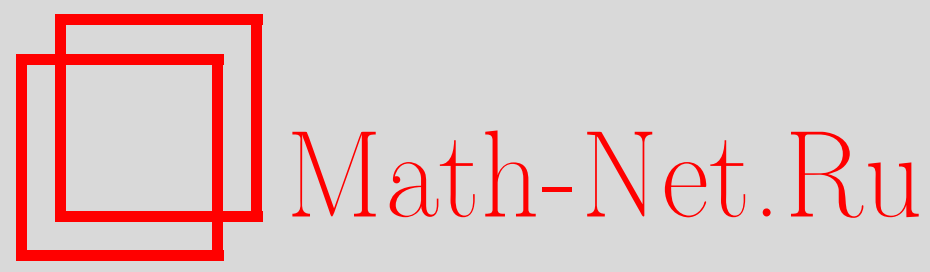

В. М. Климкин, Т. А. Срибная, Сходимость последовательности слабо регулярных функций множества, Матем. заметки, 1997, том 62, выпуск 1, 103-110

DOI: https://doi.org/10.4213/mzm1592

Использование Общероссийского математического портала Math-Net.Ru подразумевает, что вы прочитали и согласны с пользовательским соглашением http://www . mathnet.ru/rus/agreement

Параметры загрузки:

IP: 34.229 .45 .116

26 апреля 2023 г., 08:37:36

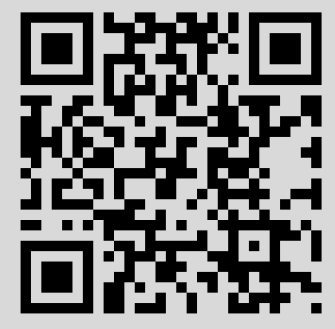




\title{
СХОДИМОСТЬ ПОСЛЕДОВАТЕЛЬНОСТИ \\ СЛАБО РЕГУЛЯРНЫХ ФУНКЦИЙ МНОЖЕСТВА
}

\author{
В. М. Климкин, Т. А. Срибная
}

\begin{abstract}
Данная работа посвящена обобщению теоремы Дьедонне о сохранении сходимости последовательности регулярных борелевских мер при переходе от системы открытых множеств компактного метрического пространства на класс всех борелевских множеств этого пространства.

Теорема Дьедонне доказана для случая, когда функции множества слабо регулярны, неаддитивны, заданы на некоторой алгебре множеств, содержащей класс открытых множеств произвольного $\sigma$-топологического пространства, и принимают значения в равномерном пространстве

Библиография: 8 названий.
\end{abstract}

\section{Известна следующая}

Теорема (Дьедонне [1]). Последовательность регулярных борелевских мер, сходящаяся на системе открытых множсеств компактного метрического пространства, сходится на всех борелевских мнохествах әтого пространства.

Эта теорема обобщалась многими авторами. На случай локально компактного пространства результат Дьедонне был распространен А. Гротендиком [2], на случай регулярного хаусдорфова пространства - П. Генсслером [3].

В предлагаемой работе теорема Дьедонне доказана для случая, когда функции множества слабо регулярны, неаддитивны, заданы на некоторой алгебре множеств, содержащей класс открытых множеств произвольного $\sigma$-топологического пространства, и принимают значения в равномерном пространстве.

Пусть $T$ - некоторое множество, $\eta$ - класс подмножеств множества $T$, замкнутьй относительно счетньх соединений и конечньх пересечений, $\varnothing \in \eta, T \in \eta$.

Следуя А. Д. Александрову [4], пространство $(T, \eta)$ будем называть $\sigma$-топологическим, множества из $\eta$ - открытымми, а их дополнения - замкнутыми.

Пусть $\mathscr{F}$ и $\mathscr{L}$ - некоторые классы (не обязательно всех) замкнутых множеств. Будем говорить, что классы множеств $\mathscr{F}$ и $\mathscr{L}$ п-отделимы, если для любых непересекающихся множеств $F \in \mathscr{F}$ и $L \in \mathscr{L}$ существуют такие открытые непересекающиеся множества $U, V \in \eta$, что $F \subset U, L \subset V$.

ПРИМЕР. Пусть $(T, \eta)$ - одно из указанных в приводимой ниже таблице топологических пространств, $\mathscr{F}$ - соответствующий класс множеств. Пусть $\mathscr{L}$ - класс всех замкнутых множеств данного пространства. Тогда пространство $(T, \eta)$ является $\sigma$-топологическим, а классы множеств $\mathscr{F}$ и $\mathscr{L} \eta$-отделимы. 


\begin{tabular}{|l|l|}
\hline \multicolumn{1}{|c|}{$(T, \eta)$} & \multicolumn{1}{|c|}{$\mathscr{F}$} \\
\hline Регулярное пространство & Класс замкнутых компактных множеств \\
Регулярное хаусдорфово пространство & Класс компактных множеств \\
Нормальное пространство & Класс замкнутых множеств \\
\hline
\end{tabular}

Пусть $\Sigma \supset \eta$-некоторая алгебра подмножеств множества $T,(S, w)$ - равномерное пространство и $\Phi=\{\varphi\}, \varphi: \Sigma \rightarrow S, \varphi(\varnothing)=e, e \in S,-$ некоторое семейство функций множества.

Если $\varphi \in \Phi, E \subset T$, то положим $\widetilde{\varphi}(E)=\{\varphi(F), F \subset E, F \in \Sigma\}$.

Обозначим через $\tau(w)$ топологию, соответствующую равномерности $w$, через $\mathscr{U}-$ фундаментальную систему окрестностей точки $e \in S$.

Будем говорить, что функция множества $\varphi \in \Phi$

- слабо $\mathscr{F}$-регулярная, если для любого множества $E \in \Sigma$ и для любого окружения $W \in w$ существует такое множество $F \in \mathscr{F}, F \subset E$, что $(\varphi(E \backslash F), e) \in W$;

- исчерпывающая на $\eta$, если для любого спектра (спектром назьвают последовательность попарно непересекающихся множеств) $\left\{U_{n}\right\} \subset \eta$

$$
\lim _{n \rightarrow \infty} \varphi\left(U_{n}\right)=e .
$$

Будем говорить, что функции множества семейства $\Phi=\{\varphi\}$

- равномерное исчерпывающее на $\eta$, если для любого спектра $\left\{U_{n}\right\} \subset \eta$ соотношение (1) вьполняется равномерно относительно $\varphi \in \Phi$;

- равномерно s-внешние, если для любого окружения $W \in w$ существует такое окружение $V \in w$, что для любой функции $\varphi \in \Phi$ и для любых непересекающихся множества $A, B \in \Sigma$ справедливо: если $(\varphi(A), e) \in V$, то $(\varphi(A \cup B), \varphi(B)) \in W$.

Заметим, что если функции множества семейства $\Phi=\{\varphi\}, \varphi: \Sigma \rightarrow(S, w)$, равномерно $s$-внешние, то для любой окрестности $u \in \mathscr{U}$ существует такая окрестность $v=v(u)$, $v \in \mathscr{U}$, что для любой функции $\varphi \in \Phi$ и для любых непересекающихся множеств $A$, $B \in \Sigma$, справедливы условия:

$$
\begin{aligned}
& \text { если } \varphi(A) \in v, \varphi(B) \in v, \text { то } \varphi(A \cup B) \in u ; \\
& \text { если } \varphi(A) \in v, \varphi(A \cup B) \in v, \text { то } \varphi(B) \in u .
\end{aligned}
$$

Если $u \in \mathscr{U}$, то всюду в дальнейшем через $\left\{u_{n}\right\} \subset \mathscr{U}$ будем обозначать такую последовательность окрестностей, что

$$
u_{1}=u \cap v(u), \quad u_{n+1}=u_{n} \cap v\left(u_{n}\right), \quad n \in \mathbb{N} .
$$

Теорема 1. Пусть $(T, \eta)$ - б-топологическое пространство, $\Sigma$ - некоторая

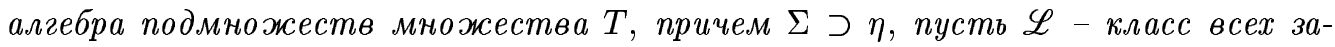
мкнутых множеств, $\mathscr{F} \subset \mathscr{L}$ и классы множсеств $\mathscr{F}$ и $\mathscr{L}$ п-отделимы. Пусть $\Phi=\left\{\varphi_{n}\right\}-$ последовательность слабо $\mathscr{F}$-регулярных, равномерно s-внешних функиий мнохества, заданных на алгебре $\Sigma \supset \eta$ и принимающих значения в отделимом равномерном пространстве $(S, w)$, причем для любого множества $H \in \eta$ последовательность $\left\{\varphi_{n}(H)\right\}$ фундаментальная. Если функиии множсества последовательности $\left\{\varphi_{n}\right\}$ равномерно исчерпывающие на системе открытых множеств $\eta$, то для любого мнохсества $E \in \Sigma$ последовательность $\left\{\varphi_{n}(E)\right\}$ фундаментальная.

Доказательство теоремы 1 опирается на следуюшие леммы, которые формулируются в предположении, что вьполнены условия теоремы. 
ЛЕмма 1. 1. Если $\varphi \in \Phi$, то для любого открытого мнохсества $H \in \eta$ и любой окрестности $u \in \mathscr{U}$, для которых $\widetilde{\varphi}(H) \not \subset u$, существует открытое мнохество $A \in \eta$ такое, что $A \subset H, \varphi(A) \notin u_{1}$.

2. Если $\varphi \in \Phi$, то для любого множсества $E \in \Sigma$ и любой окрестности $и \in \mathscr{U}$, для которых $\widetilde{\varphi}(E) \not \subset u$, существует замкнутое множсество $F \in \mathscr{F}$ такое, что $F \subset E, \varphi(F) \notin u_{1}$.

ДокаЗАтЕЛьство. 1. Пусть множество $H \in \eta$ и окрестность $u \in \mathscr{U}$ такие, что $\widetilde{\varphi}(H) \not \subset u$. Тогда существует множество $E \in \Sigma$, такое, что $E \subset H, \varphi(E) \notin u$. Так как функция $\varphi$ слабо $\mathscr{F}$-регулярная, то существует такое множество $C \in \mathscr{F}$, что $C \subset H \backslash E$, $\varphi((H \backslash E) \backslash C) \in u_{1}$.

Положим $A=H \backslash C$. Из $(2)$ и равенства $A=(A \backslash E) \cup E$ следует, что множество $A$ искомое.

Пункт 2 доказьвается аналогично.

Лемма 2. Для любой окрестности $u \in \mathscr{U}$ и любого множсества $F \in \mathscr{L}$ существует такое открытое мнохсество $H \in \eta$, что $F \subset H, \widetilde{\varphi}(H \backslash F) \subset u$ для любой функиии $\varphi \in \Phi$.

ДокАЗАТЕЛЬСтво. Предположим противное. Тогда существуют окрестность $u \in \mathscr{U}$ и множество $F_{0} \in \mathscr{L}$ такие, что для любого открытого множества $H \supset F_{0}$ найдется такая функция $\varphi \in \Phi$, для которой $\widetilde{\varphi}\left(H \backslash F_{0}\right) \not \subset u$.

По предположению существует такая функция $\varphi_{n_{1}} \in \Phi$, что $\widetilde{\varphi}_{n_{1}}\left(T \backslash F_{0}\right) \not \subset u$.

В силу п. 2 леммы 1 существует такое множество $F_{1} \in \mathscr{F}$, что

$$
F_{1} \subset T \backslash F_{0}, \quad \varphi_{n_{1}}\left(F_{1}\right) \notin u_{1} .
$$

Так как классы множество $\mathscr{F}$ и $\mathscr{L} \eta$-отделимы, то существуют такие открытые множества $A_{1}^{\prime}$ и $A_{2}^{\prime}$, что

$$
F_{0} \subset A_{1}^{\prime}, \quad F_{1} \subset A_{2}^{\prime}, \quad A_{1}^{\prime} \cap A_{2}^{\prime}=\varnothing .
$$

В силу (3), (4) и п. 1 леммы 1 существует такое открытое множество $A_{1} \subset A_{2}^{\prime}$, что

$$
\varphi_{n_{1}}\left(A_{1}\right) \notin u_{2} \text {. }
$$

По предположению для открытого множества $A_{1}^{\prime}$ существует такая функция $\varphi_{n_{2}} \in \Phi$, что

$$
\widetilde{\varphi}_{n_{2}}\left(A_{1}^{\prime} \backslash F_{0}\right) \not \subset u .
$$

В силу п. 2 леммы 1 существует такое множество $F_{2} \in \mathscr{F}$, что

$$
F_{2} \subset A_{1}^{\prime} \backslash F_{0}, \quad \varphi_{n_{2}}\left(F_{2}\right) \notin u_{1} .
$$

Так как классы множеств $\mathscr{F}$ и $\mathscr{L} \quad \eta$-отделимы, то существуют такие открытые множества $A_{1}^{\prime \prime}$ и $A_{2}^{\prime \prime}$, что

$$
F_{0} \subset A_{1}^{\prime \prime} \subset A_{1}^{\prime}, \quad F_{2} \subset A_{2}^{\prime \prime} \subset A_{1}^{\prime}, \quad A_{1}^{\prime \prime} \cap A_{2}^{\prime \prime}=\varnothing .
$$

В силу (5), (6) и п. 1 леммы 1 сушествует такое открытое множество $A_{2} \subset A_{2}^{\prime \prime}$, что

$$
\varphi_{n_{2}}\left(A_{2}\right) \notin u_{2} \text {. }
$$

Продолжив процесс по индукции, построим спектр $\left\{A_{k}\right\}$ открытых множеств и подпоследовательность $\left\{\varphi_{n_{k}}\right\} \subset \Phi$, для которых

$$
\varphi_{n_{k}}\left(A_{k}\right) \notin u_{2}, \quad k \in \mathbb{N},
$$

что противоречит равномерной исчерпьваемости на $\eta$ функций множества последовательности $\left\{\varphi_{n}\right\}$. 
Лемма 3. Для любой окрестности $u \in \mathscr{U}$ и любого множества $E \in \Sigma$ существует такой конечный набор множеств $F_{1}, \ldots, F_{n}$ из $\mathscr{F}$, что

$$
F_{i} \subset E, \quad i=\overline{1, n}, \quad \widetilde{\varphi}\left(E \backslash \bigcup_{i=1}^{n} F_{i}\right) \subset u
$$

для любой функиии $\varphi \in \Phi$.

ДокАЗАТЕЛЬСтво. Предположим противное. Тогда существуют окрестность $u \in \mathscr{U}$ и множество $E_{0} \in \Sigma$ такие, что для любого конечного набора множеств $F_{1}, \ldots, F_{n} \in \mathscr{F}$, $F_{i} \subset E_{0}, i=\overline{1, n}$, существует функция $\varphi \in \Phi$, для которой

$$
\tilde{\varphi}\left(E_{0} \backslash \bigcup_{i=1}^{n} F_{i}\right) \not \subset u .
$$

По предположению существует такая функция $\varphi_{n_{1}} \in \Phi$, что

$$
\widetilde{\varphi}_{n_{1}}\left(E_{0}\right) \not \subset u \text {. }
$$

В силу п. 2 леммы 1 существует такое множество $F_{1} \in \mathscr{F}$, что

$$
F_{1} \subset E_{0}, \quad \varphi_{n_{1}}\left(F_{1}\right) \notin u_{1} .
$$

По предположению существует функция $\varphi_{n_{2}} \in \Phi$, для которой

$$
\widetilde{\varphi}_{n_{2}}\left(E_{0} \backslash F_{1}\right) \not \subset u \text {. }
$$

В силу п. 2 леммы 1 найдется такое множество $F_{2} \in \mathscr{F}$, что

$$
F_{2} \subset E_{0} \backslash F_{1}, \quad \varphi_{n_{2}}\left(F_{2}\right) \notin u_{1}
$$

Продолжив процесс по индукции, построим спектр $\left\{F_{k}\right\} \subset \mathscr{F}, F_{k} \subset E_{0}, k \in \mathbb{N}$, и последовательность функций $\left\{\varphi_{n_{k}}\right\} \subset \Phi$ такие, что

$$
\varphi_{n_{k}}\left(F_{k}\right) \notin u_{1}, \quad k \in \mathbb{N} .
$$

Для окрестности $u_{2} \in \mathscr{U}$ и спектра $\left\{F_{n}\right\} \subset \mathscr{F}$ построим последовательность множеств $\left\{V_{n}\right\} \subset \eta$ и спектр $\left\{d_{n}\right\} \subset \eta$ такие, что

$$
\begin{gathered}
F_{n} \subset V_{n}, \quad d_{n} \subset V_{n}, \\
\widetilde{\varphi}\left(V_{n} \backslash d_{n}\right) \subset u_{2}, \quad n \in \mathbb{N},
\end{gathered}
$$

для любой функции $\varphi \in \Phi$.

В силу леммы 2 для любого множества $F_{n} \in \mathscr{F}, n \in \mathbb{N}$, существует множество $H_{n} \in \eta$ такое, что

$$
F_{n} \subset H_{n}, \quad \widetilde{\varphi}\left(H_{n} \backslash F_{n}\right) \subset u_{n+2}
$$

для любой функции $\varphi \in \Phi$. 
Так как классы множеств $\mathscr{F}$ и $\mathscr{L} \eta$-отделимы, то существуют такие множества $V_{n} \in \eta$ и $L_{n} \in \mathscr{L}$, что

$$
F_{n} \subset V_{n} \subset L_{n} \subset H_{n}, \quad n \in \mathbb{N} \text {. }
$$

Положим

$$
d_{n}=V_{n} \backslash \bigcup_{i=0}^{n-1} L_{i}, \quad n \in \mathbb{N}, \quad L_{0}=\varnothing
$$

Из соотношения

$$
V_{n} \backslash d_{n} \subset \bigcup_{i=1}^{n}\left(H_{i} \backslash F_{i}\right), \quad n \in \mathbb{N},
$$

в силу (2) и (10) получаем

$$
\widetilde{\varphi}\left(V_{n} \backslash d_{n}\right) \subset u_{2}
$$

для любой функции $\varphi \in \Phi$.

Из (7) и (8) следует, что

$$
\widetilde{\varphi}_{n_{k}}\left(V_{k}\right) \not \subset u_{1}, \quad k \in \mathbb{N} \text {. }
$$

Из (9) и (11) следует, что

$$
\widetilde{\varphi}_{n_{k}}\left(d_{k}\right) \not \subset u_{2}, \quad k \in \mathbb{N} .
$$

В силу п. 1 леммы 1 существуют открытые множества $A_{k} \subset d_{k}$, для которых

$$
\varphi_{n_{k}}\left(A_{k}\right) \notin u_{3}, \quad k \in \mathbb{N},
$$

что противоречит равномерной исчерпьваемости на $\eta$ функций множества последовательности $\left\{\varphi_{n}\right\}$.

ДоКАЗАТЕЛЬСТво ТЕОРЕмЫ 1. Покажем сначала, что для любой окрестности $u \in \mathscr{U}$ и для любого множества $E \in \Sigma$ существует такое открытое множество $H \in \eta$, что

$$
\widetilde{\varphi}_{n}(E \triangle H) \subset u, \quad n \in \mathbb{N} .
$$

Пусть $u \in \mathscr{U}, E \in \Sigma$. По лемме 3 существует такое замкнутое множество $F \in \mathscr{L}$, что $F \subset Е$ и

$$
\widetilde{\varphi}_{n}(E \backslash F) \subset u_{1}, \quad n \in \mathbb{N} .
$$

Согласно лемме 2 существует такое открытое множество $H \in \eta$, что $F \subset H$ и

$$
\widetilde{\varphi}_{n}(H \backslash F) \subset u_{1}, \quad n \in \mathbb{N} \text {. }
$$

Из (13) и (14) следует, что

$$
\widetilde{\varphi}_{n}(E \triangle H) \subset u, \quad n \in \mathbb{N} \text {. }
$$

Покажем теперь, что для любого множества $E \in \Sigma$ последовательность $\left\{\varphi_{n}(E)\right\}$ фундаментальная.

Возьмем из $w$ произвольное окружение $W$, симметричное окружение $W_{1}$ такое, что $W_{1} \circ W_{1} \circ W_{1} \subset W$, симметричное окружение $W_{2}$ такое, что $W_{2} \circ W_{2} \subset W_{1}$, и, наконец, окружение $W_{3}$, которое находится для окружения $W_{2}$ из того условия, что функции множества последовательности $\left\{\varphi_{n}\right\}$ равномерно $s$-внешние. 
Тогда в силу (12) для любого $E \in \Sigma$ существует такое открытое множество $H \in \eta$, что

$$
\widetilde{\varphi}_{n}(E \triangle H) \subset\left\{y \in S:(e, y) \in W_{3}\right\}, \quad n \in \mathbb{N} .
$$

Отсюда следует, что $\left(\varphi_{n}(E), \varphi_{n}(E \cap H)\right) \in W_{2}$,

$$
\left(\varphi_{n}(E \cap H), \varphi_{n}(H)\right) \in W_{2}, \quad\left(\varphi_{n}(E), \varphi_{n}(H)\right) \in W_{1} .
$$

Так как последовательность $\left\{\varphi_{n}(H)\right\}$ фундаментальная, то существует номер $n_{0}$ такой, что для всех $m, n>n_{0}$

$$
\left(\varphi_{n}(H), \varphi_{m}(H)\right) \in W_{1} .
$$

Отсюда и из (15) получим, что для всех $m, n>n_{0}$

$$
\left(\varphi_{n}(E), \varphi_{m}(E)\right) \in W
$$

что и требовалось доказать.

Tеорема 2. Пусть $(T, \eta), \Sigma, \mathscr{L}$ u $\mathscr{F}$ - me жсе, что и в теореме 1. Пусть $(S, w)$ - отделимое равномерное пространство; $\varphi_{n}: \Sigma \rightarrow(S, w), n \in \mathbb{N},-$ слабо $\mathscr{F}$-регулярные, равномерно s-внешние функиии множества, каждая из которых исчерпывающая на $\eta$. Пусть $\varphi_{0}: \Sigma \rightarrow S-$ исчерпывающая на $\eta$ функиия множества, причем для любого мнохества $H \in \eta$

$$
\lim _{n \rightarrow \infty} \varphi_{n}(H)=\varphi_{0}(H)
$$

Тогда для любого множества $E \in \Sigma$

$$
\lim _{n \rightarrow \infty} \varphi_{n}(E)=\varphi_{0}(E) .
$$

ДокАЗАТЕЛЬСТво. В силу теоремы 1 достаточно показать, что функции множества последовательности $\left\{\varphi_{n}\right\}$ равномерно исчерпывающие на $\eta$. Предположим противное. Тогда существуют окрестность $u \in \mathscr{U}$ и спектр $\left\{H_{n}\right\} \subset \eta$, для которых

$$
\varphi_{n}\left(H_{n}\right) \notin u, \quad n \in \mathbb{N} \text {. }
$$

Так как функция множества $\varphi_{0}$ исчерпьвающая на $\eta$, то существует подспектр $\left\{H_{n_{i}}\right\} \subset\left\{H_{n}\right\}$, такой, что

$$
\widetilde{\varphi}_{0}\left(\bigcup_{i=1}^{\infty} H_{n_{i}}\right) \in u_{2} .
$$

Без ограничения общности можно считать, что

$$
\widetilde{\varphi}_{0}\left(\bigcup_{n=1}^{\infty} H_{n}\right) \in u_{2} .
$$

Пусть $n_{1}=1$. В силу (16) и (18) существует такой номер $n_{2}>n_{1}$, что $\varphi_{n_{2}}\left(H_{n_{1}}\right) \in u_{2}$. Аналогично найдем номер $n_{3}>n_{2}$ такой, что

$$
\left\{\varphi_{n_{3}}\left(H_{n_{1}}\right), \varphi_{n_{3}}\left(H_{n_{2}}\right), \varphi_{n_{3}}\left(H_{n_{1}} \cup H_{n_{2}}\right)\right\} \subset u_{2}
$$


Продолжив процесс по индукции, построим такую последовательность номеров $\left\{n_{k}\right\}$, что

$$
\left\{\varphi_{n_{k}}\left(\bigcup_{i \in J} H_{n_{i}}\right), J \subset \overline{1, k-1}\right\} \subset u_{2}, \quad k=2,3, \ldots
$$

Без ограничения общности можно считать, что

$$
\left\{\varphi_{k}\left(\bigcup_{i \in J} H_{i}\right), J \subset \overline{1, k-1}\right\} \subset u_{2}, \quad k=2,3, \ldots
$$

Из (2), (17) и (19) следует, что

$$
\varphi_{k}\left(\left(\bigcup_{i \in J} H_{i}\right) \cup H_{k}\right) \notin u_{1}, \quad J \subset \overline{1, k-1}, \quad k=2,3, \ldots
$$

Так как функции множества $\varphi_{n}, n \in \mathbb{N}$, исчерпывающие на $\eta$, то существует подспектр $\left\{H_{k_{i}}\right\} \subset\left\{H_{k}\right\}$ такой, что

$$
\widetilde{\varphi}_{k_{i}}\left(H_{k_{i+1}} \cup H_{k_{i+2}} \cup \cdots\right) \in u_{2}
$$

Из (20) следует, что

$$
\varphi_{k_{i}}\left(H_{k_{1}} \cup \cdots \cup H_{k_{i}}\right) \notin u_{1}, \quad i=2,3, \ldots
$$

Из (21) и (22) следует, что

$$
\varphi_{k_{i}}\left(\bigcup_{i=1}^{\infty} H_{k_{i}}\right) \notin u_{2}, \quad i=2,3, \ldots
$$

А из (16) и (18) следует, что существует номер $i_{0}$, такой, что для всех $i>i_{0}$ справедливо

$$
\varphi_{k_{i}}\left(\bigcup_{i=1}^{\infty} H_{k_{i}}\right) \in u_{2}
$$

Полученное противоречие завершает доказательство теоремы.

ЗАмЕчАниЕ 1. Примерами равномерно $s$-внешних функций множества являются семейства $k$-внешних мер [5, с. 913], семейства квазилипшицевых функций множества [6, c. 26], семейства квазиаддитивных многозначных функций множества [7, с. 38].

Следовательно, для каждого из этих семейств функций множества справедливы результаты теорем 1 и 2.

Приведем два таких следствия.

Пусть $G$ - топологическая абелева группа, $\mathscr{H}$ - фундаментальная система окрестностей нуля в $G$. Функция множества $\varphi: \Sigma \rightarrow G$ назьвается $k$-внешней мерой, $k \in \mathbb{N}$, если для любой окрестности $u \in \mathscr{H}$ и любых непересекаюшихся множеств $A, B \in \Sigma$ справедливо: если $\varphi(A) \in u$, то $\varphi(A \cup B)-\varphi(B) \in k \cdot u$, где $k \cdot u=\left\{\alpha_{1}+\cdots+\alpha_{k}, \alpha_{i} \in u\right.$, $i=\overline{1, k}\}$. 
СлЕДСТвИЕ 1. Пусть $(T, \eta), \Sigma, \mathscr{L}$ и $\mathscr{F}$ - me же, что в теореме $1, G$ - топологическая абелева группа, $\varphi_{n}: \Sigma \rightarrow G, n \in \mathbb{N},-$ последовательность $k$-внешних мер.

Если функиии множества последовательности $\left\{\varphi_{n}\right\}$ слабо $\mathscr{F}$-регулярные, равномерно исчерпывающие на $\eta$ и для любого множсества $H \in \eta$ последовательность $\left\{\varphi_{n}(H)\right\}$ фундаментальная, то для любого мнохсества $E \in \Sigma$ последовательность $\left\{\varphi_{n}(E)\right\}$ фундаментальная.

СлЕДСтвиЕ 2. Пусть $(T, \eta), \Sigma, \mathscr{L}$ u $\mathscr{F}$ те же, что и в теореме $1, G$ - топологическая абелева группа, $\varphi_{n}: \Sigma \rightarrow G, n \in \mathbb{N},-$ последовательность слабо $\mathscr{F}$-регулярных, аддитивных, исчерпывающих на

Если для любого мнохества $H \in \eta$ последовательность $\left\{\varphi_{n}(H)\right\}$ фундаментальная, то для любого мнохества $E \in \Sigma$ последовательность $\left\{\varphi_{n}(E)\right\}$ фундаментальная.

ДокАЗАТЕЛЬСТво. В силу [8, следствие 2.4] функции множества последовательности $\left\{\varphi_{n}\right\}$ равномерно исчерпьвающие на $\eta$. Так как аддитивные функции множества являются равномерно $s$-внешними, то утверждение следствия вытекает из теоремы 1 .

ЗАмЕчАниЕ 2. Очевидно, что следствие 2 останется справедливым, если вместо аддитивных, исчерпывающих на $\eta$ функций множества $\varphi_{n}: \Sigma \rightarrow G, n \in \mathbb{N}$, рассматривать счетно аддитивные функции множества.

\section{СПИСОК ЦИТИРОВАННОЙ ЛИТЕРАТУРЫ}

[1] Dieudonné J. Sur la convergence des suites de mesures de Radon // An. Acad. Brasil. Ciênc. 1951. V. 23. P. 21-38, 277-282.

[2] Grothendieck A. Sur les applications linéaires faiblement compactes d'espaces du type $C(K)$ // Canad. J. Math. 1953. V. 51. P. 129-173.

[3] Gänssler P. A convergence theorem for measures in regular Hausdorff spaces // Math. Scand. 1971. V. 29. P. 237-244.

[4] Александров А. Д. Аддитивные функции множества в абстрактных пространствах // Матем. сб. 1941. Т. 9(51). С. 563-628.

[5] Саженков А.Н. Ограниченность векторных мер // Матем. заметки. 1979. Т. 25. №6. C. $913-917$.

[6] Гусельников Н. С. О продолжении квазилипшицевых функций множества // Матем. заметки. 1975. Т. 17. № 1. С. 21-31.

[7] Drewnowski L. Additive and countable additive correspondences // Rocz. Pol. Tow. Mat. Ser. I. 1976. V. 19. №1. P. 25-54.

[8] Lucia P., Morales P. Some consequences of the Brooks-Jeweff theorem for additive uniform semigroup-valued functions // Confer. Sem. Mat. Univ. Bari. 1988. V. 227. P. 1-23. 